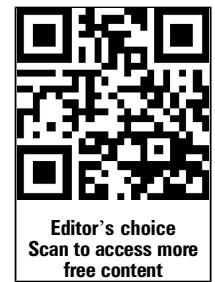

- Additional material is published online only. To view please visit the journal online (http://dx.doi.org/10.1136/ jmedgenet-2013-101637)

For numbered affiliations see end of article.

Correspondence to Dr Peristera Paschou, Department of Molecular Biology and Genetics, Democritus University of Thrace, Alexandroupoli 68100, Greece,

ppaschou@mbg.duth.gr

Received 5 March 2013 Revised 8 May 2013 Accepted 27 May 2013 Published Online First 3 July 2013

To cite: Karagiannidis I, Dehning $S$, Sandor $P$, et al. J Med Genet 2013;50. 760-764.

\title{
Support of the histaminergic hypothesis in Tourette Syndrome: association of the histamine decarboxylase gene in a large sample of families
}

\author{
Iordanis Karagiannidis, ${ }^{1}$ Sandra Dehning, ${ }^{2}$ Paul Sandor, ${ }^{3}$ Zsanett Tarnok, \\ Renata Rizzo, ${ }^{5}$ Tomasz Wolanczyk, ${ }^{6}$ Marcos Madruga-Garrido, ${ }^{7}$ \\ Johannes Hebebrand, ${ }^{8}$ Markus M Nöthen, ${ }^{9}$ Gerd Lehmkuhl, ${ }^{10}$ Luca Farkas, ${ }_{1}^{4}$ \\ Peter Nagy, ${ }^{4}$ Urszula Szymanska, ${ }^{6}$ Zachos Anastasiou, ${ }^{1}$ Vasileios Stathias, ${ }^{1}$ \\ Christos Androutsos, ${ }_{11}^{11}$ Vaia Tsironi, ${ }^{11}$ Anastasia Koumoula, ${ }^{11}$ Csaba Barta, ${ }^{12}$ \\ Peter Zill, ${ }^{2}$ Pablo Mir, ${ }^{7,13}$ Norbert Müller, ${ }^{2}$ Cathy Barr, ${ }^{3}$ Peristera Paschou ${ }^{1}$
}

\section{ABSTRACT}

Background Gilles de la Tourette Syndrome is a neurodevelopmental disorder that is caused by the interaction of environment with a complex genetic background. The genetic etiology of the disorder remains, so far, elusive, although multiple promising leads have been recently reported. The recent implication of the histamine decarboxylase $(H D C)$ gene, the key enzyme in histamine production, raises the intriguing hypothesis of a possible role of histaminergic dysfunction leading to TS onset.

Methods Following up on the finding of a nonsense mutation in a single family with TS, we investigated variation across the HDC gene for association with TS. As a result of a collaborative international effort, we studied a large sample of 520 nuclear families originating from seven European populations (Greek, Hungarian, Italian, Polish, German, Albanian, Spanish) as well as a sample collected in Canada.

Results and Conclusions Interrogating 12 tagging SNPs (tSNP) across the HDC region, we find strong over-transmission of alleles at two SNPs (rs854150 and rs 1894236) in the complete sample, as well as a statistically significant associated haplotypes. Analysis of individual populations also reveals signals of association in the Canadian, German and Italian samples. Our results provide strong support for the histaminergic hypothesis in TS etiology and point to a possible role of histamine pathways in neuronal development.

\section{INTRODUCTION}

Gilles de la Tourette Syndrome (TS) is a multifactorial neurodevelopmental disorder resulting from the complex interaction of environment with genetic backround. TS is characterised by the appearance of multiple motor and vocal tics, and high comorbidity rates with other neuropsychiatric disorders of childhood such as obsessive compulsive disorder (OCD), attention deficit hyperactivity disorder (ADHD) and autism, suggesting the possibility of a common aetiological background. Due to lack of education of health professionals and the general public, TS remains poorly understood and severely underdiagnosed in many countries despite the fact that recent investigations have reported a prevalence of $0.4-1 \%$ in populations of European origin. ${ }^{1}$ Results from large-scale studies and collaborative efforts have only very recently started to become available (eg, refs. 2-4) and the genetic basis of the disorder remains, so far, elusive, although multiple chromosomal regions have been implicated, providing several promising leads that warrant further investigation. ${ }^{5}$

Tics are thought to result from dysfunctions in cortical and subcortical regions that are involved in habit formation, including the basal ganglia and related cortical and thalamic structures. ${ }^{6}$ TS has traditionally been viewed as a disorder of dopaminergic neurotransmission; however, other transmitters and neuromodulators have also been implicated. ${ }^{6}$ In fact, the recent implication of the L-histidine decarboxylase (HDC) gene in TS aetiology has raised the intriguing hypothesis of the involvement of histaminergic neural pathways in the onset of the disorder. ${ }^{7} H D C$ is highly conserved throughout different species and catalyses the oxidative decarboxylation of histidine to histamine. ${ }^{8}$ Ercan-Sencicek et $a l^{7}$ studied a family with one father and eight of his children affected with TS, and found a linkage signal on chromosome 15. Sequencing of all genes within the linked interval led to the discovery of a single rare coding mutation, a premature termination codon (p. W317*, c. $951 \mathrm{G}>\mathrm{A}$ ) in exon 9 of the HDC gene. The mutation could not be found in any of the 3000 control individuals of Western European origin who were screened. At the same time, resequencing of the coding region of HDC in 720 patients with TS and 360 controls revealed no additional nonsense variants, demonstrating the fact that the nonsense mutation identified in the index family is extremely rare.

Since the original study that implicated HDC in TS aetiology, Lei et $a l^{9}$ screened the HDC gene for exonic mutations in 100 Chinese Han patients with TS, and could only find three variants, not predicted to result in amino acid changes: a $\mathrm{C}>\mathrm{T}$ intronic transition (IVS1 $+52 \mathrm{C}>\mathrm{T}$ ), which did not affect the splicing site, a synonymous $\mathrm{C}>\mathrm{A}$ transition (c.426C $>\mathrm{A}$ ) in exon 4 and a synonymous $\mathrm{G}>\mathrm{A}$ transition (c.1743G $>\mathrm{A}$ ) in exon 12. ${ }^{9}$ The first genomewide association study for TS only produced a weak association signal close to the HDC 
gene ( $\mathrm{p}$ value of 0.02 with rs7166052). ${ }^{3}$ However, a recent genomewide scan for de novo or transmitted rare CNVs in TS found enrichment of genes within histamine receptor signalling pathways. ${ }^{10}$ Furthermore, a genomewide study of 95 French Canadian trios with familial history of $\mathrm{TS}^{11}$ showed association to a chromosome 15 microsatellite marker (D15S1016) that lies within the same interval found to be linked with TS in the original study that implicated the HDC gene in TS aetiology. ${ }^{7}$

Following up on the novel hypothesis of the involvement of HDC and histaminergic neural pathways in TS aetiology, we investigated the possible association of variation across the HDC gene in a large sample of families originating from Canada as well as multiple countries in Europe. We find significant association to the HDC gene in our sample, providing strong support for the role of histamine in the genesis and mediation of tics, and pointing to new lines of research in this area.

\section{MATERIAL AND METHODS}

\section{Genetic association study samples}

Five independently collected samples of TS nuclear families were analysed (total of 520 families). Details of age of onset per sample, sex distribution as well as comorbid OCD and ADHD are shown in online supplementary table S1. The Tourette Syndrome Genetics-Southern and Eastern Europe Initiative sample included European-descent families of Polish (36 trios), Italian (50 trios), Hungarian (84 trios), Greek (10 trios) and Albanian origin (6 trios). Assessment was performed by on-site clinicians using the tools provided by the TS Association International Consortium for Genetics. ${ }^{2}$ TS was ascertained according to DSM-IV-TR criteria for Italy, Hungary, Albania and Greece, and DSM-IV for Poland. Our study also included two independent samples of German descent. The first was the German Collaborative TS Research Group sample (96 trios), collected using DSM-III-R criteria. ${ }^{12}{ }^{13}$ Our second sample of German ancestry was collected in Munich (69 trios), using DSM-IV-TR criteria. Our fourth European sample was of Spanish ancestry (19 families), collected using DSM-IV-TR criteria. Finally, we also analysed a large sample of TS trios collected in Canada (150 families), using DSM-III-R criteria. Differences between DSM-III-R, DSM-IV and DSM-IV-TR are minimal; the upper age limit of onset is 18 in DSM-IV (and
DSM-IV-TR) and 21 in DSM-III-R, and the 'marked distress' criterion, possibly pointing to more severe cases, only appears in DSM-IV (applied only to the Polish families). ${ }^{14}$ For all samples, collection was approved by local Ethics Boards and informed consent was taken from all participating individuals or their parents.

\section{Selection of single nucleotide polymorphisms, genotyping and statistical analysis}

Using the HapMap CEPH European population as reference, we selected tagging single nucleotide polymorphisms (tSNPs) in order to capture variation for a $40 \mathrm{~kb}$ region including the $\mathrm{HDC}$ gene. A total of $12 \mathrm{tSNPs}$ were selected (see online supplementary table S2) capturing variation at an additional nine SNPs with a mean $\mathrm{r}^{2}$ of 0.968 . All selected SNPs are intronic. Genotyping was performed using the KASP genotyping method, a competitive allele-specific assay based on Fluorescent Resonance Energy Transfer from LGC Genomics and Kbioscience (http://www. lgcgenomics.com). The average genotyping success was greater than $99 \%$. The linkage disequilibrium (LD) structure of each gene in the different studied populations was analysed using Haploview. ${ }^{15}$ In order to test for association of the studied SNPs with the TS phenotype, the transmission test for linkage disequilibrium (TDT) was performed, as also implemented in Haploview. ${ }^{15}$ We report here association results on single SNPs as well as SNP haplotypes of haplotype blocks defined with the Gabriel et $\mathrm{al}^{16} \mathrm{cri}^{-}$ teria (tests with one degree of freedom, as implemented by Haploview). In order to correct for multiple comparisons, 1000 permutations were performed (using Haploview) and the adjusted $\mathrm{p}$ values are also reported here (table 1).

\section{RESULTS}

Genotyping results for all SNPs conformed to Hardy-Weinberg equilibrium proportions and Mendelian transmission within families. Rare allele frequencies for each analysed sample are shown in online supplementary figure S1. Since the transmission disequilibrium test (TDT) is robust to population stratification, we were able to perform analysis for the complete sample that was available to us, as well as families within each individual population. Single SNP analysis for association with the TS phenotype in the complete sample (520 families) produced

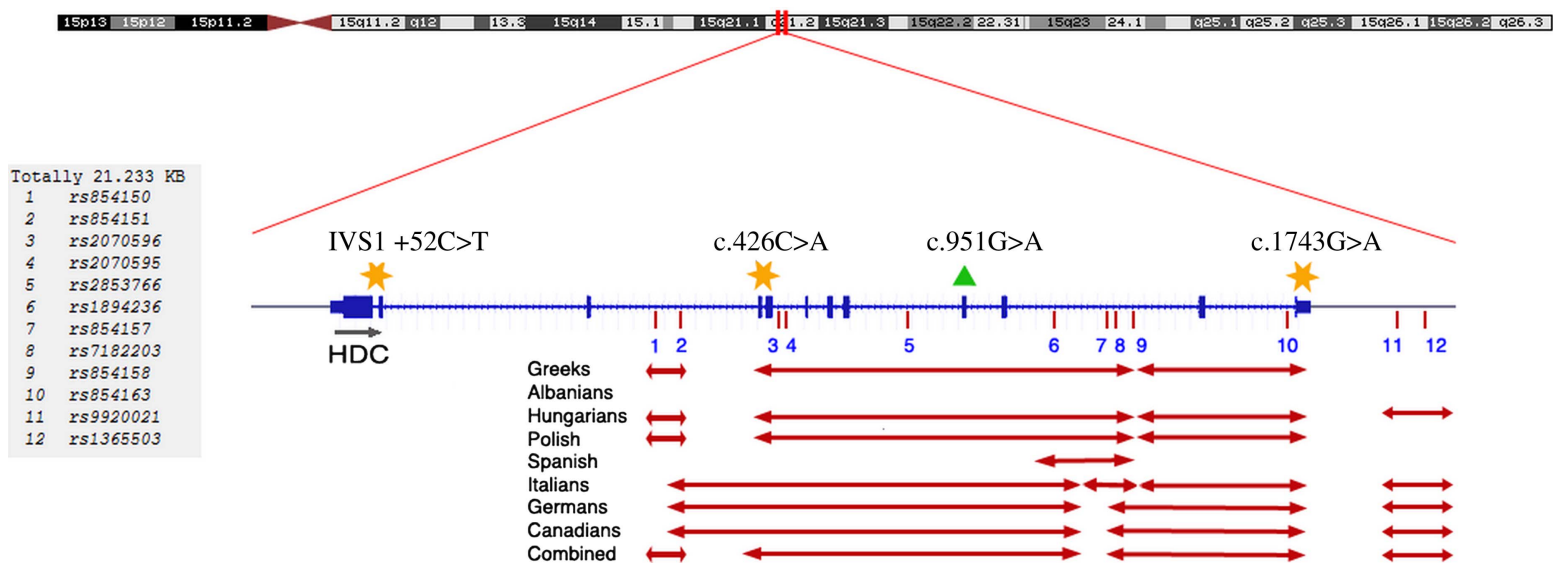

Figure 1 Linkage disequilibrium structure of studied region covering the L-histidine decarboxylase (HDC) gene (chromosome 15: 48 316 000$48356000 \mathrm{NCBI}$ build 36). Unrelated individuals from a sample of 520 nuclear families were analysed using Haploview and haplotype blocks were defined using the Gabriel et $a^{16}$ criteria and visualised with the software Haplot. HDC variants found in individuals with Gilles de la Tourette Syndrome (TS) in previous studies are also shown. The triangle indicates the position of the originally described mutation that led to a premature termination codon ( $p$. W $317^{*}, c .951 \mathrm{G}>\mathrm{A}$ ) in a family with $\mathrm{TS}^{7}{ }^{7}$ and the stars indicate the position of variants described in Chinese Han patients with TS (not predicted to result in amino acid changes). ${ }^{9}$ Access the article online to view this figure in colour. 
Table 1 Transmission test for linkage disequilibrium for L-histidine decarboxylase variation tSNPs and haplotypes

\begin{tabular}{|c|c|c|c|c|c|}
\hline Rs\# & Over-transmitted allele & $\mathrm{T}: \mathrm{U}$ & $\chi^{2}$ & $\mathrm{p}$ Value & Permutation $p$ value \\
\hline rs854150 & G & $284: 220$ & 8.127 & 0.0044 & 0.0290 \\
\hline rs854151 & G & $205: 190$ & 0.57 & 0.4504 & \\
\hline rs2070596 & $\mathrm{T}$ & $192: 154$ & 4.173 & 0.0411 & 0.2640 \\
\hline rs2070595 & G & $232: 224$ & 0.14 & 0.7079 & \\
\hline rs 2853766 & $A$ & $215: 174$ & 4.321 & 0.0376 & 0.2370 \\
\hline rs1894236 & C & $201: 143$ & 9.779 & 0.0018 & 0.0150 \\
\hline rs854157 & $T$ & $277: 245$ & 1.962 & 0.1613 & \\
\hline rs7182203 & G & $217: 215$ & 0.009 & 0.9233 & \\
\hline rs854158 & G & $246: 216$ & 1.948 & 0.1628 & \\
\hline rs854163 & $A$ & $223: 188$ & 2.981 & 0.0843 & \\
\hline rs9920021 & $A$ & $227: 198$ & 1.979 & 0.1595 & \\
\hline rs1365503 & A & $249: 223$ & 1.432 & 0.2314 & \\
\hline Haplotype & Frequency & $T: U$ & $x^{2}$ & $\mathrm{p}$ Value & Permutation $p$ value \\
\hline \multicolumn{6}{|l|}{ Block 1} \\
\hline CA & 0.620 & $224.5: 286.7$ & 7.56 & 0.006 & 0.0140 \\
\hline GG & 0.268 & $227.2: 195.0$ & 2.465 & 0.1164 & \\
\hline GA & 0.107 & $123.3: 87.1$ & 6.212 & 0.0127 & 0.0440 \\
\hline \multicolumn{6}{|l|}{ Block 2} \\
\hline TGGC & 0.387 & $243.0: 273.2$ & 1.761 & 0.1845 & \\
\hline TGAC & 0.248 & $218.0: 173.0$ & 5.179 & 0.0229 & 0.0790 \\
\hline ATGT & 0.216 & $151.0: 198.9$ & 6.561 & 0.0104 & 0.0420 \\
\hline TTGC & 0.140 & $147.5: 122.3$ & 2.346 & 0.1256 & \\
\hline
\end{tabular}

strong results of overtransmission of alleles for SNP rs854150 and rs1894236 (table 1). These results remained statistically significant after performing 1000 permutations of the data. Using the Gabriel et $a l^{16}$ criteria for the combined sample, four haplotype blocks were defined across the studied region. The first of the two TS-associated SNPs in our analysis (rs854150) resides within a two-SNP haplotype block, which is also significantly associated with the TS phenotype, both for the protective and the susceptibility alleles (table 1). Significant association is also found with the haplotype block that carries the second TS-associated SNP in our analysis (rs1894236). This is a five-SNP haplotype spanning $9.2 \mathrm{~Kb}$ (SNPs rs854151, rs2070596, rs2070595, rs2853766 and rs1894236). The rs 1894236 allele that is overtransmitted to TS patients is found on four different haplotypes. On the other hand, the undertransmitted, and thus, protective allele, is only found on a single haplotype at a frequency of $0.21 \%$, which is also significantly undertransmitted to TS patients (permutation $\mathrm{p}=0.04)($ table 1 ).

Individual population analysis also revealed interesting results that further support a possible role for HDC in TS aetiology (see online supplementary tables S3 and S4). In our combined sample of German origin (our largest individual population sample with 165 trios), SNP rs854150 retained a trend of overtransmission to TS patients (uncorrected $p$ value of 0.04 ), which, however, did not withstand permutation testing. Nevertheless, an additional association signal was found, including the haplotype block of the two SNPs most distal to our studied region (rs9920021 and rs1365503) with a permutation $\mathrm{p}$ value of 0.048 . Interestingly, in our Canadian sample (150 families), these two same SNPs produced the strongest signals of association with permutation $\mathrm{p}$ values of 0.001 for $\mathrm{rs} 1365503$ and 0.021 for rs9920021. SNP rs1894236 was also significantly associated with TS in the Canadian sample. In the Italian population (50 trios), SNP rs7182203 was associated with the TS phenotype (permutation $\mathrm{p}$ value of 0.043 ), as well as a threesite haplotype including this SNP as well as rs854158 and rs854163. Individual analysis of the remaining samples did not produce any remarkable results, which could be due to small individual sample size. We should also note that analysis of the Canadian, German and Italian samples revealed an identical haplotype block structure, while a slightly different structure was observed for the remaining populations that we analysed.

\section{DISCUSSION}

Following up on the recent evidence about the involvement of the HDC gene and histaminergic neural pathways in the aetiology of TS, we studied variation across HDC for association with TS in a large sample of 520 nuclear families from seven European populations (Greece, Hungary, Poland, Italy, Albania, Germany and Spain) as well as a sample collected in Canada. We employed a tSNPs approach and identified strong overtransmission of alleles at two SNPs (rs854150 and rs1894236). Strong association was also found with a five-SNP haplotype carrying the protective rs1894236 allele, as well as two-SNP haplotypes carrying rs854150. Both SNPs that were found to be associated with TS in our study reside in intronic regions of HDC. However, it is worth noting that rs1894236 lies under one of the Encyclopedia of DNA Elements (ENCODE) project peaks for an enhancer-associated histone mark (UCSC genome browser NCBI36/hg18, http:/genome.ucsc.edu). The ENCODE project has systematically mapped regions of transcription, transcription factor association, chromatin structure and histone modification, and histone marks are specific chemical modifications in histone sequence which may, for instance, enhance gene 
transcription. ${ }^{17}$ Thus, this particular region may be directly involved in regulation of transcription of the HDC gene. Individual population analysis produced additional signals of positive association. Thus, our results strongly support the histaminergic hypothesis for TS development.

Histamine is an evolutionary conserved signalling molecule that plays a central role in gastric acid secretion, innate and acquired immunity and immunomodulation, bronchoconstriction, vasodilation and neurotransmission. The neuronal histaminergic system is involved in a number of basic physiological functions, such as circadian rhythmicity, energy metabolism, neuroendocrine homeostasis, stress, sensory and motor functions, cognition, attention, learning and memory. ${ }^{18}$ Neuronal histamine is exclusively synthesised in the tuberomamillary nucleus of the hypothalamus (TMN), while histamine-positive fibres have been described projecting to cortex, hippocampus, basal ganglia, thalamus and virtually all other regions of the central nervous system. ${ }^{18}$ HDC is the key enzyme for histamine production from histidine. In the brain, its mRNA is expressed exclusively in the posterior hypothalamus. The specific regulation of HDC gene expression in the brain is not understood. However, neuroactive peptides and steroids are among the factors that control HDC transcription and degradation in various tissues and contexts (eg, oxidative stress). ${ }^{8}{ }^{18}$ Recently, diurnal variation in the levels of HDC mRNA in the TMN has been observed in agreement with a role of neuronal histamine in regulating day-night patterns. ${ }^{19}$

Histamine binds to and acts through four known G proteincoupled receptors ( $\mathrm{H} 1$ through $\mathrm{H} 4)$ and a polyamine binding site on glutamatergic N-Methyl-D-Aspartate (NMDA) receptors. ${ }^{18}$ High densities of $\mathrm{H} 2 \mathrm{R}$ and H3R are found in the basal ganglia, ${ }^{18} 20$ especially on the principal neurons of the striatum, the GABAergic medium spiny neurons (MSN). ${ }^{18}{ }^{21}$ Furthermore, H3R mRNAs in the cortex and in the substantia nigra pars compacta indicate the presence of $\mathrm{H} 3$ heteroreceptors on the major inputs to the striatum. ${ }^{20}$ Thus, histaminergic innervations of anatomical structures that have been related to TS has been confirmed. Furthermore, H3R regulates a variety of neurotransmitters, including dopamine and serotonin. On the other hand, $H d c-/-$ deficient mice have several traits relevant to features of TS and have shown decreased brain histamine and increased sensitivity to stereotypic behaviours upon administration of dopamine agonists. ${ }^{22}$ Such stimulant-induced movements, including rearing, sniffing and biting have previously been proposed as a model of human tics. ${ }^{23}$

So far, the histaminergic system has not received as much attention as other monoaminergic systems of the brain. Classically established as a 'peripherally' important mediator of inflammation, the importance of histamine in neurotransmission and its role in neuropsychiatric disorders are only recently starting to be appreciated. For instance, it has been found severely affected in age-related neurodegenerative diseases such as Parkinson disease (PD) and Alzheimer disease (AD), while histamine receptors are becoming a prime target for pharmaceutical research and development and a number of agents have entered into clinical assessment as cognition enhancers for ADHD, schizophrenia, $\mathrm{AD}$ and PD. ${ }^{24}$ Adding to the multiple facets of neural histamine, our findings further support the implication of histaminergic dysfunction in TS aetiology, providing new insight. An interesting next step could be the functional characterisation of RNA transcripts in samples that carry the associated HDC genetic variants in order to help elucidate their role and clarify the possible involvement of histamine pathways in neurodevelopmental disorders.

\section{Author affiliations}

${ }^{1}$ Department of Molecular Biology and Genetics, Democritus University of Thrace, Alexandroupoli, Greece

${ }^{2}$ Department of Psychiatry, Ludwig-Maximilians-University Munich, Munich, Germany ${ }^{3}$ Toronto Western Research Institute, University Health Network, Toronto, Ontario,

Canada

${ }^{4}$ Vadaskert Clinic for Child and Adolescent Psychiatry, Budapest, Hungary

${ }^{5}$ Materno-Infantile and Radiological Science Department, University of Catania, Catania, Italy

${ }^{6}$ Department of Child Psychiatry, Medical University of Warsaw, Warsaw, Poland ${ }^{7}$ Unidad de Trastornos del Movimiento, Servicio de Neurología y Neurofisiología Clínica/Sección de Neuropediatría, Instituto de Biomedicina de Sevilla, Hospital Universitario Virgen del Rocío/CSIC/Universidad de Sevilla, Seville, Spain

${ }^{8}$ Department of Child and Adolescent Psychiatry, University of Duisburg-Essen, Essen, Germany

${ }^{9}$ Department of Genomics, Life and Brain Center, Institute for Human Genetics, University of Bonn, Bonn, Germany

${ }^{10}$ Department of Child and Adolescent, Psychiatry of the University of Cologne, Cologne, Germany

${ }^{11}$ Child and Adolescent Psychiatry Clinic, Sismanoglio General Hospital of Attica, Athens, Greece

${ }^{12}$ Department of Medical Chemistry, Molecular Biology and Pathobiochemistry, Semmelweis University, Budapest, Hungary

${ }^{13}$ Centro de Investigación Biomédica en Red sobre Enfermedades Neurodegenerativas (CIBERNED), Seville, Spain

Acknowledgements This study was made possible thanks to the collaborative efforts of Tourette Syndrome Genetics - Southern and Eastern Europe Initiative (TSGeneSEE) and COST Action BM905: European Network for the Study of GTS (EUNETGTS). We would like to acknowledge the following members of these consortia who have also contributed in this work with sample collection (in alphabetical order by country): Mira Kapisyzi, Entela Basha (University Hospita Center 'Mother Theresa', Tirana, Albania); Inmaculada Bernal-Bernal, Marta Bonilla-Toribio, Fátima Carrillo, María T Cáceres-Redond, Marta Correa-Vela Ismael Huertas-Fernández, Laura Vargas-González (Unidad de Trastornos del Movimiento, Servicio de Neurología y Neurofisiología Clínica/Sección de Neuropediatría. Instituto de Biomedicina de Sevilla, Hospital Universitario Virgen del Rocío/CSIC/Universidad de Sevilla, Seville, Spain), Pilar Gómez-Garre (Unidad de Trastornos del Movimiento, Servicio de Neurología y Neurofisiología Clínica/Sección de Neuropediatría. Instituto de Biomedicina de Sevilla, Hospital Universitario Virgen del Rocío/CSIC/Universidad de Sevilla, Seville, Spain; Centro de Investigación Biomédica en Red sobre Enfermedades Neurodegenerativas, Spain).

Contributors IK performed experiments and wrote the paper. SD contributed samples, participated in the design of experiments and analysis and in manuscript preparation. PS contributed samples, participated in the design of experiments and analysis and in manuscript preparation. ZT contributed samples, participated in the design of experiments and analysis and in manuscript preparation. RR contributed samples, participated in the design of experiments and analysis and in manuscript preparation. TW contributed samples, participated in the design of experiments and analysis and in manuscript preparation. MM contributed samples, participated in the design of experiments and analysis and in manuscript preparation. JH contributed samples, participated in the design of experiments and analysis and in manuscript preparation. MMN contributed samples, participated in the design of experiments and analysis and in manuscript preparation. GL contributed samples, participated in the design of experiments and analysis and in manuscript preparation. LF contributed samples, participated in the design of experiments and analysis and in manuscript preparation. PN contributed samples, participated in the design of experiments and analysis and in manuscript preparation. US contributed samples, participated in the design of experiments and analysis and in manuscript preparation. ZA performed experiments and analysis. Vassilios Stathias performed experiments and analysis. CA contributed samples, participated in the design of experiments and analysis and in manuscript preparation. VT contributed samples, participated in the design of experiments and analysis and in manuscript preparation. AK contributed samples, participated in the design of experiments and analysis and in manuscript preparation. CB contributed samples, participated in the design of experiments and analysis and in manuscript preparation. PZ participated in the design of experiments and analysis and in manuscript preparation. PM contributed samples, participated in the design of experiments and analysis and in manuscript preparation. NM contributed samples, participated in the design of experiments and analysis and in manuscript preparation. CB contributed samples, participated in the design of experiments and analysis and in manuscript preparation. PP conceived the study, designed experiments and analysis and prepared the manuscript.

Funding This work was supported by COST Action BM0905 (http://tourette-eu.org) and Tourette Syndrome Association, USA Research Grant Awards (2008-2010).

Competing interests None.

Ethics approval Research Ethics Committee, Democritus University of Thrace. 
Provenance and peer review Not commissioned; externally peer reviewed.

Data sharing statement All data are available upon request to the authors and the TSGeneSEE consortium.

\section{REFERENCES}

1 Robertson MM. The prevalence and epidemiology of Gilles de la Tourette syndrome. Part 1: the epidemiological and prevalence studies. J Psychosom Res 2008;65:461-72.

2 Tourette Syndrome Association International Consortium for Genetics. Genome scan for Tourette disorder in affected-sibling-pair and multigenerational families. Am J Hum Genet 2007;80:265-72.

3 Scharf JM, Yu D, Mathews CA, Neale BM, Stewart SE, Fagerness JA, Evans P, Gamazon E, Edlund CK, Service SK, Tikhomirov A, Osiecki L, Illmann C, Pluzhnikov A, Konkashbaev A, Davis LK, Han B, Crane J, Moorjani P, Crenshaw AT, Parkin MA, Reus VI, Lowe TL, Rangel-Lugo M, Chouinard S, Dion Y, Girard S, Cath DC, Smit JH, King RA, Fernandez TV, Leckman JF, Kidd KK, Kidd JR, Pakstis AJ, State MW, Herrera LD, Romero R, Fournier E, Sandor P, Barr CL, Phan N, Gross-Tsur V, Benarroch F, Pollak Y, Budman CL, Bruun RD, Erenberg G, Naarden AL, Lee PC, Weiss N, Kremeyer B, Berrío GB, Campbell DD, Silgado JC Cardona, Ochoa WC, Restrepo SC Mesa, Muller H, Duarte AV Valencia, Lyon GJ, Leppert M, Morgan J, Weiss R, Grados MA, Anderson K, Davarya S, Singer H, Walkup J, Jankovic J, Tischfield JA, Heiman GA, Gilbert DL, Hoekstra PJ, Robertson MM, Kurlan R, Liu C, Gibbs JR, Singleton A; for the North American Brain Expression Consortium, Hardy J; for the UK Human Brain Expression Database, Strengman E, Ophoff RA, Wagner M, Moessner R, Mirel DB, Posthuma D, Sabatti C, Eskin E, Conti DV, Knowles JA, Ruiz-Linares A, Rouleau GA, Purcell S, Heutink P, Oostra BA, McMahon WM, Freimer NB, Cox NJ, Pauls DL. Genome-wide association study of Tourette's syndrome. Mol Psychiatry 2013;18:721-8.

4 Karagiannidis I, Rizzo R, Tarnok Z, Wolanczyk T, Hebebrand J, Nöthen MM, Lehmkuhl G, Farkas L, Nagy P, Barta C, Szymanska U, Panteloglou G, Miranda DM, Feng Y, Sandor P, Barr C; TSGeneSEE, Paschou P. Replication of association between a SLITRK1 haplotype and Tourette Syndrome in a large sample of families. Mol Psychiatr 2012;17:665-8.

5 Paschou P. The genetic basis of Gilles de la Tourette Syndrome. Neurosci Biobehav Rev 2013;37:1026-39.

6 Felling RJ, Singer HS. Neurobiology of tourette syndrome: current status and need for further investigation. J Neurosci 2011;31:12387-95.

7 Ercan-Sencicek AG, Stillman AA, Ghosh AK, Bilguvar K, O'Roak BJ, Mason CE, Abbott T, Gupta A, King RA, Pauls DL, Tischfield JA, Heiman GA, Singer HS, Gilbert DL, Hoekstra PJ, Morgan TM, Loring E, Yasuno K, Fernandez T, Sanders S, Louvi A, Cho JH, Mane S, Colangelo CM, Biederer T, Lifton RP, Gunel M, State MW. L-histidine decarboxylase and Tourette's syndrome. N Eng/ J Med 2010;362:1901-8.

8 Fleming JV, Fajardo I, Langlois MR, Sanchez-Jimenez F, Wang TC. The C-terminus of ratL-histidine decarboxylase specif-ically inhibits enzymic activity and disrupts pyridoxal phosphate-dependent interactions with L-histidine substrate analogues. Biochem J 2004;381:769-78.

9 Lei J, Deng X, Zhang J, Su L, Xu H, Liang H, Huang X, Song Z, Deng H. Mutation screening of the HDC gene in Chinese Han patients with Tourette syndrome. Am J Med Genet Part B 2012;159B:72-6.
10 Fernandez TV, Sanders SJ, Yurkiewicz IR, Ercan-Sencicek AG, Kim YS, Fishman DO, Raubeson MJ, Song Y, Yasuno K, Ho WS, Bilguvar K, Glessner J, Chu SH, Leckman JF, King RA, Gilbert DL, Heiman GA, Tischfield JA, Hoekstra PJ, Devlin B, Hakonarson H, Mane SM, Günel M, State MW. Rare copy number variants in tourette syndrome disrupt genes in histaminergic pathways and overlap with autism. Biol Psychiatry 2012;71:392-402.

11 Rivière JB, St-Onge J, Gaspar $C$, Diab S, Dion $Y$, Lespérance $P$, Tellier $G$, Richer F, Chouinard S, Dubé MP, Rouleau GA; Montreal Tourette Study Group. Genome-wide TDT analysis in French-Canadian families with Tourette syndrome. Can J Neurol Sci 2010:37:110-2

12 Hebebrand J, Klug B, Fimmers R, Seuchter SA, Wettke-Schäfer R, Deget F, Camps A, Lisch S, Hebebrand K, von Gontard A, Lehmkuhl G, Poustka F, Schmidt M, Baur MP, Remschmidt $H$. Rates for tic disorders and obsessive compulsive symptomatology in families of children and adolescents with Gilles de la Tourette syndrome. J Psychiatr Res 1997;31:519-30.

13 Schoenian S, Konig I, Oertel W, Remschmidt H, Ziegler A, Hebebrand J, Bandmann O. HLA-DRB genotyping in Gilles de la Tourette patients and their parents. American Journal of Medical Genetics Part B: Neuropsychiatric Genetics 2003;119B:60-4.

14 Cath DC, Hedderly T, Ludolph AG, Stern JS, Murphy T, Hartmann A, Czernecki V, Robertson MM, Martino D, Munchau A, Rizzo R; ESSTS Guidelines Group. European clinical guidelines for Tourette syndrome and other tic disorders. Part I: assessment. Eur Child Adolesc Psychiatry 2011;20:155-71.

15 Barrett JC, Fry B, Maller J, Daly MJ. Haploview: analysis and visualization of LD and haplotype maps. Bioinformatics 2005;21:263-5.

16 Gabriel SB, Schaffner SF, Nguyen H, Moore JM, Roy J, Blumenstiel B, Higgins J, DeFelice M, Lochner A, Faggart M, Liu-Cordero SN, Rotimi C, Adeyemo A, Cooper R, Ward R, Lander ES, Daly MJ, Altshuler D. The structure of haplotype blocks in the human genome. Science 2002;296:2225-9.

17 ENCODE Project Consortium. An integrated encyclopedia of DNA elements in the human genome. Nature 2012;489:57-74.

18 Haas HL, Sergeeva OA, Selbach O. Histamine in the nervous system. Physiol Rev 2008;88:1183-241.

19 Shan L, Bossers K, Unmehopa U, Bao AM, Swaab DF. Alterations in the histaminergic system in Alzheimer's disease: a postmortem study. Neurobiol Aging 2012;33:2585-98.

20 Pillot C, Heron A, Cochois V, Tardivel-Lacombe J, Ligneau X, Schwartz JC, Arrang JM. A detailed mapping of the histamine $\mathrm{H}(3)$ receptor and its gene transcripts in rat brain. Neuroscience 2002;114:173-93.

21 Goodchild RE, Court JA, Hobson I, Piggott MA, Perry RH, Ince P, Jaros E, Perry EK. Distribution of histamine $\mathrm{H} 3$-receptor binding in the normal human basal ganglia: comparison with Huntington's and Parkinson's disease cases. Eur J Neurosci 1999;11:449-56.

22 Kubota Y, Ito C, Sakurai E, Sakurai E, Watanabe T, Ohtsu H. Increased methamphetamine-induced locomotor activity and behavioral sensitization in histamine-deficient mice. J Neurochem 2002;83:837-45.

23 Saka E, Graybiel AM. Pathophysiology of Tourette's syndrome: striatal pathways revisited. Brain Dev 2003;25(Suppl 1):S15-9.

24 Passani MB, Blandina P. Histamine receptors in the CNS as targets for therapeutic intervention. Trends Pharmacol Sci 2011;32:242-9. 